\title{
Upper threshold of burst duration for continuous apparent motion - Effects of amplitude modulation and frequency band-
}

\author{
Ikuichiro Kinoshita and Shigeaki Aoki \\ NTT Cyber Space Laboratories, \\ 3-9-11 Midori-cho, Musashino, 180-8585 Japan
}

(Received 4 December 1997)

\begin{abstract}
The upper thresholds of burst duration that produced continuous apparent motion were measured when stimuli were successively presented using three loudspeakers arranged in the horizontal plane. Stimuli tested were broadband noises with amplitude modulation, tones and bandpass noises. The upper threshold depended on stimulus and tended to be longer than that of $72 \mathrm{~ms}$ for an unmodulated broadband noise. For amplitude-modulated broadband noises, there was an increase in the upper threshold from 72 to $290 \mathrm{~ms}$ as the modulation index increased from 0.0 to 1.0. However, the upper threshold decreased from 360 to $135 \mathrm{~ms}$ with an increase in modulation frequency from 4 to $64 \mathrm{~Hz}$. For tones, the upper threshold displayed a concave function of frequency with a minimum of $71 \mathrm{~ms}$ at a frequency of $3 \mathrm{kHz}$. For bandpass noises, the upper threshold fell from 347 to $101 \mathrm{~ms}$ as the bandwidth broadened from $1 / 12$ to 3.0 octaves. These results are discussed in connection with perception for amplitude modulation and mechanisms to detect motion.
\end{abstract}

Keywords: Hearing, Apparent motion, Burst duration, Amplitude modulation, Frequency, Bandwidth

PACS number: 43. 66. Mk, 43. 66. Qp

\section{INTRODUCTION}

Apparent motion is an illusion that can be produced by successively presenting stimuli located in distinct positions. It is considered to be a general sensory phenomenon, since it can be observed not only in the auditory modality but also in the visual and tactual modalities. Successive presentation may be the simplest way of simulating a motion by using stationary stimuli with appropriate timing and location.

The primary factors responsible for auditory apparent motion are the burst duration per stimulus and the interstimulus onset interval (ISOI). Based on the relationship between the burst duration and the ISOI, Briggs and Perrott (1972) classified the perception under dichotic listening into five categories : single sound, simultaneous sounds, continu- ous motion, broken motion, and successive sounds. Strybel et al. (1989, 1990 and 1992) also investigated the relationship between the category and the timing under binaural and monaural conditions in a free field. They replicated the results obtained by Briggs and Perrott (1972). The perception of continuous motion is often referred to as optimal motion in other literatures (see, for example, Korte, 1915 ; Burtt, 1917 ; Scholz, 1924 ; Hisata, 1934 etc.).

Continuous motion usually breaks down when there are silent gaps between the stimulus presentations, or the burst duration is shorter than the ISOI. When the burst duration is equal to the ISOI, the perception of successively presented stimuli varies with an increase of the burst duration, as follows: (1) When the burst duration is short enough (about $10 \mathrm{~ms}$ ), an unmoving single sound or simultaneous sounds are heard. (2) When the burst duration is 
longer ( 20 to $70 \mathrm{~ms}$ ), a continuous moving motion is heard. (3) At even longer burst durations (70 to $100 \mathrm{~ms}$ ), broken motion is perceived; then, (4) at burst durations exceeding $100 \mathrm{~ms}$, successive sounds are perceived. Thus, there is an upper threshold of burst duration for continuous apparent motion.

The range of burst duration and the ISOI for auditory apparent motion is not sensitive to the location and the spatial interval of stimuli (Nakajima et al., 1990; Strybel et al., 1990; Strybel et al., 1992; Strybel 1993; Mizushima et al., 1994), unlike that for visual apparent motion (Korte, 1915; Scholz, 1924; etc.). The timing condition for auditory continuous motion is also independent of whether monaural listening or binaural listening is used (Strybel and Neale, 1994). Even when the interval between stimuli is within the minimum audible angle (MAA) or auditory acuity, motion can be heard (Strybel and Neale, 1994).

Another aspect of apparent motion has been investigated from the standpoint of the temporal variation in amplitude, by evaluating the detectability of silent gaps among stimuli. According to Mizushima et al. (1994), the threshold for detecting silent gap using two spatially separated stimuli with a broadband noise was less than $2 \mathrm{~ms}$. This threshold is nearly equal to the threshold for detecting silent gap using identical stimuli (Forrest and Green, 1987). The coincidence between the threshold to detect silent gap for spatially separated stimuli and the threshold for identical stimuli was also observed by using one-octave band of noise (Nakanishi et al., 1994; Shailer and Moore, 1983). Under those conditions, the threshold was $6 \mathrm{~ms}$. Nakanishi et al. attributed this factor of the increase in the threshold to the fluctuation in the stimuli.

The hypothesis that temporal variation in amplitude is used as a cue to motion (see, for example, Strybel and Neale, 1994) is supported by the abovecited properties of apparent motion, and is consistent with the following findings: (1) a stimulus presented from an unmoving sound driver can be perceived as motion at a certain timing of the amplitude variation (Strybel, 1994), and (2) there are neurons sensitive to amplitude modulation due to movement of stimuli (Stumpf et al., 1992).

Although these facts suggest mediation by processing of amplitude modulation for motion detection, the correlation between the position of the stimulus and the amplitude was inevitable in the procedure of the experiments. We assume that alleviating the correlation will shed light on the mechanisms for motion detection. This alleviation can be conducted by introducing amplitude modulation having a modulation period different from the burst duration.

In our recent investigation, we measured the upper threshold of burst duration for continuous apparent motion (Kinoshita and Aoki, 1993). The upper threshold differs significantly among sources and ranges from $72 \mathrm{~ms}$ for broadband noise to 580 $\mathrm{ms}$ for voice. One of the possible causes of the difference in the upper thresholds was amplitude modulation. However, the complexity of the characteristics in the stimuli tested hampers specification of the primary factors contributing to the difference of the upper threshold.

In an attempt to confirm the factor of the difference, we measured the upper threshold of burst duration for the continuous apparent motion, using the following stimuli: (1) sinusoidally amplitudemodulated broadband noises as a function of modulation index $m$ and the modulation frequency $f_{\mathrm{m}}$ with an attempt to isolate the modulation components most affecting the upper threshold, (2) tones as a function of frequency, and (3) bandpass noises as a function of bandwidth. The prospective upper thresholds may provide clues to considering the roles in motion detection by means of amplitude modulation and frequency analyses, in a different approach of Nakajima et al. (1990).

\section{EXPERIMENT}

This section describes the method and conditions we used to measure the upper threshold of burst duration for continuous apparent motion. Unless specified otherwise, we used the same setup and procedure in all experiments, which are described in sections 3.1 through 3.4 .

\subsection{Subjects}

Two women and two men (aged between 25 and 36) with normal hearing participated in the experiment. The two men were the authors of this paper. The two other subjects did not have any previous knowledge of the experiment. Before formal data were collected, each subject underwent preliminary tests to learn how to respond to the stimuli. One trial block of 120 trials was conducted for the preliminary data and two trial blocks were conduct- 


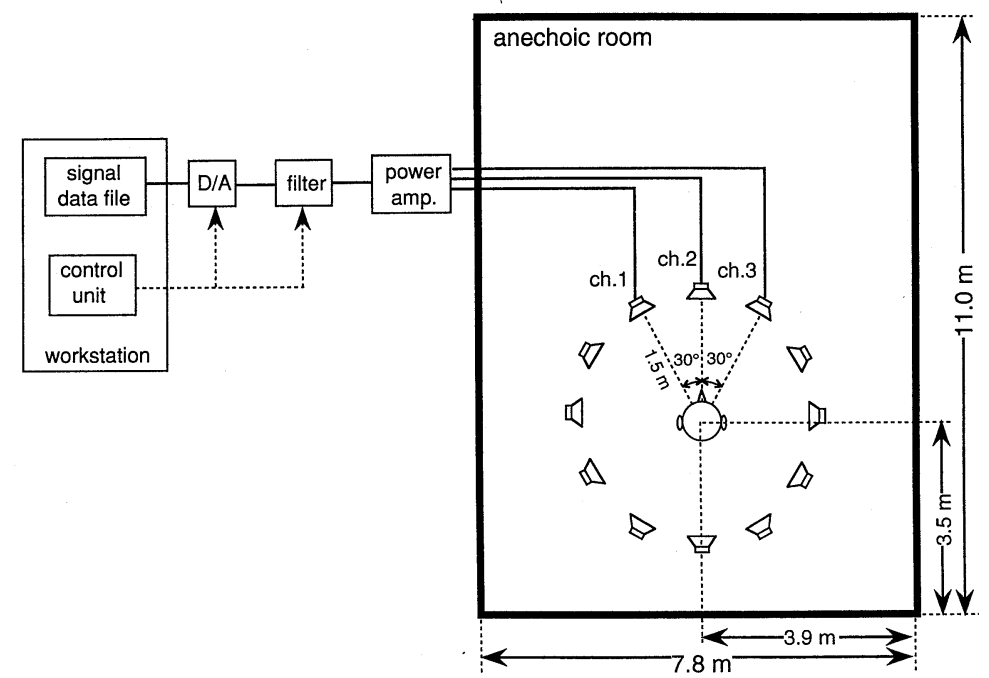

Fig. 1 Illustration of experimental setup composed of a workstation, D/A converter, filter, and power amplifier, and the location of the actuated loudspeakers and the subject in an anechoic room.

ed for the formal data. The preliminary data were discarded before estimation of the upper threshold.

\subsection{Setup}

Testing was conducted in a large anechoic room $(11.0 \times 7.8 \times 6.6 \mathrm{~m})$, the walls of which were covered with $80-\mathrm{cm}$-long acoustic wedges to reduce sound reflection. As shown in Fig. 1, a subject was seated in a round chair positioned near the center of the room and was instructed to face directly ahead without moving. The subject was not blindfolded, even though the inside of the room was visible. Twelve 12-cm diameter full-range loudspeakers (AURATONE 5PSC) were distributed in a horizontal arc ( $1.5 \mathrm{~m}$ in radius) centered at the listening point. Three of the loudspeakers were actuated: the center one was in front of the subject and the other two were positioned $30^{\circ}$ left and right of the center. Nine others were used as dummies. Except for the authors, the subjects had no previous knowledge as to which of the loudspeakers would present stimuli.

\subsection{Stimulus Generation}

The signal was generated digitally from a data file by a computer (HP-srx 835). As shown in Fig. 1, the signal was converted into an analog signal through a 16-bit digital-to-analog converter (NSG MD-9000) at a sampling rate of $24 \mathrm{kHz}$. Then it

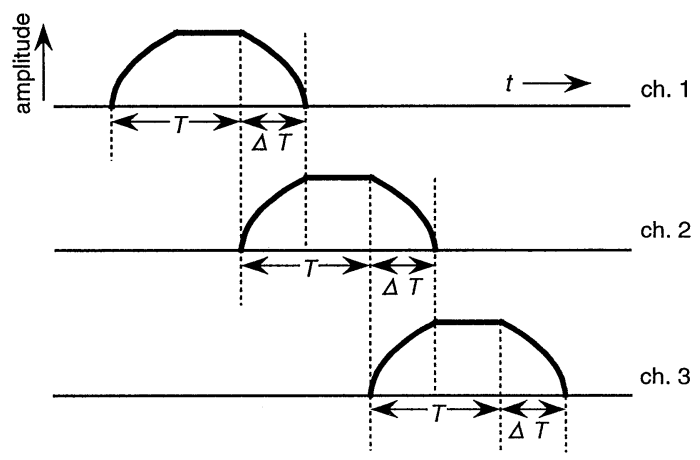

Fig. 2 Envelopes for the stimuli tested, presented in succession from ch. 1 to ch. 3 at burst duration $T$ with both rise and decay of duration $\Delta T$ of $10 \mathrm{~ms}$.

was passed through a low-pass filter with a decay of $90 \mathrm{~dB}$ per octave (NF P-86) and high-pass filters with a decay of $135 \mathrm{~dB}$ per octave (NF P-88).

The signal was switched by modifying the data file. A loudspeaker was actuated when the digital signal was assigned to the channel corresponding to that loudspeaker, while zeros were stuffed to the other channels. Since the signal was shifted for each $f_{\mathrm{s}} T$ sample in the order of the channel, stimuli were successively presented from one loudspeaker on the left or right, to the middle, and then to the loudspeaker on the opposite side for a duration $T$ 
(Fig. 2), where $f_{\mathrm{s}}$ was the sampling frequency. The signal on each channel was faded in and out sinusoidally with rise and decay times $\Delta T$ of $10 \mathrm{~ms}$. The sound level measured at the subject was $65 \mathrm{~dB}$ (A-weighted) in a free field.

\subsection{Procedure}

Each trial block in the test was conducted for each subject using a fixed token of stimuli (e.g., modulation index or modulation frequency for amplitudemodulated broadband noise). Each block consisted of 120 trials. For each trial, the burst duration was selected randomly from the following 12 durations : $40,50,60,80,100,120,150,200,250,300,400$ and $500 \mathrm{~ms}$. Ten trials were executed for each duration in a trial block. The order of stimulus presentation, whether clockwise or counterclockwise, was also randomly chosen for each trial. Thus a single condition of burst duration and presentation order was repeated in five trials per trial block. Immediately after each trial, the subject was instructed to reply by word of mouth whether the sound appeared to move continuously or not. The percentage of responses for continuous motion was collected from each subject for each burst duration. The upper threshold of the burst duration for continuous apparent motion, where the percentage of responses for continuous motion was $50 \%$, was then determined (Fig. 3).

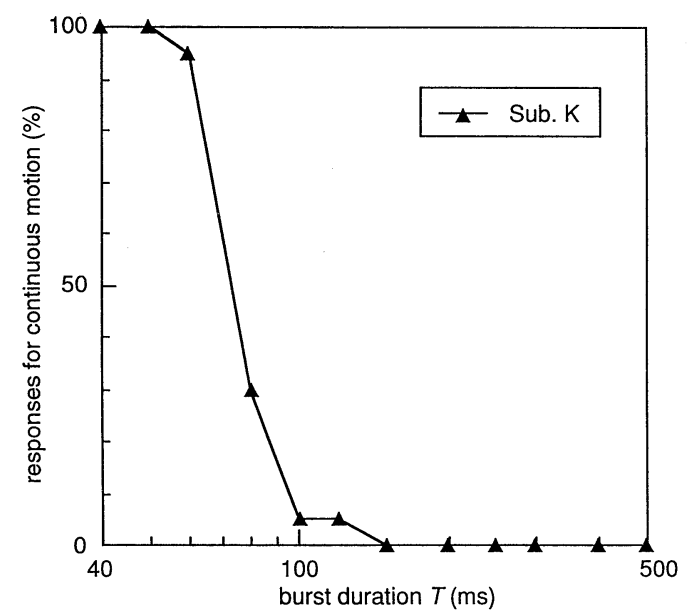

Fig. 3 An example of a psychometric function of burst duration $T$ based on responses from one subject for unmodulated broadband noise. The upper threshold was defined at $50 \%$ for continuous motion.

\section{RESULTS AND DISCUSSION}

3.1 Effect of Modulation Index for AmplitudeModulated Broadband Noise

\section{A. Stimuli}

A pseudo-random sequence generated in the computer was used for the amplitude-modulated broadband noise. The sequence was multiplied by the following sinusoidal function :

$$
1+m \sin \left(2 \pi f_{\mathrm{m}} i / f_{\mathrm{s}}\right),
$$

where $m$ is the modulation index, $f_{\mathrm{m}}$ is the modulation frequency, $f_{\mathrm{s}}$ is the sampling frequency (fixed at $24 \mathrm{kHz}$ ), and $i$ is the order in the signal, so that the sinusoidal modulation

$$
1+m \sin \left(2 \pi f_{\mathrm{m}} t\right),
$$

was introduced into the original random sequence, where $t$ is the time. The modulated signal was stored in the data file before generating the stimuli.

Modulation indices $m$ of $0,0.2,0.4,0.6,0.8$ and 1.0 were tested. The modulation frequency $f_{\mathrm{m}}$ was fixed at $16 \mathrm{~Hz}$. The cutoff frequencies for the lowand the high-pass filters were set to be $7.0 \mathrm{kHz}$ and $200 \mathrm{~Hz}$, respectively.

B. Results

Figure 4 shows the upper threshold as a function of modulation index $m$. The upper threshold increases with an increase of $m$; for example $72 \mathrm{~ms}$ for $m$ of 0.0 (no modulation) and $290 \mathrm{~ms}$ for $m$ of

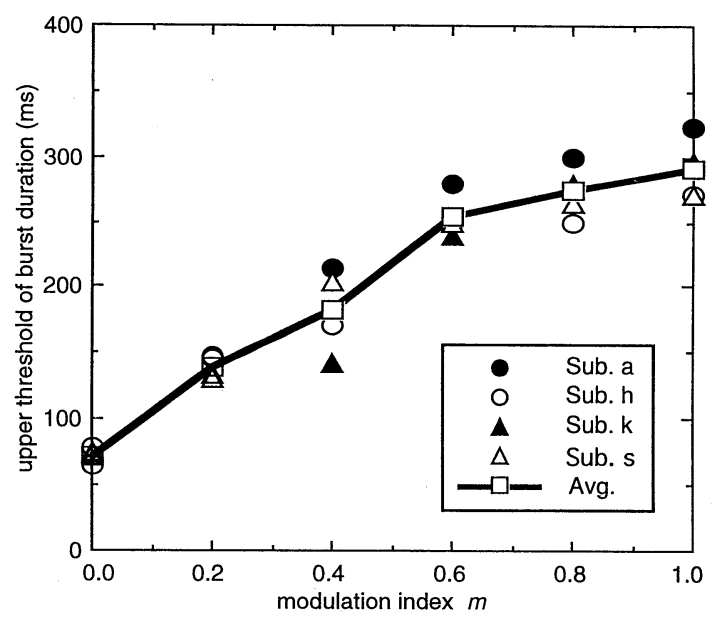

Fig. 4 Upper threshold of burst duration for amplitude-modulated broadband noises as a function of modulation index $m$ at a modulation frequency $f_{\mathrm{m}}$ of $16 \mathrm{~Hz}$. 


\section{KINOSHITA and S. AOKI : DURATION FOR CONTINUOUS APPARENT MOTION}

1.0 (full modulation). An analysis of variance on the data showed that $m$ had a significant effect on the increase in the upper threshold $(F(5,15)=$ $44.11 ; p<0.001)$, while no significant effect of subject was shown $(F(3,15)=0.393 ; p>0.1)$.

3.2 Effect of Modulation Frequency for Amplitude-Modulated Broadband Noise

\section{A. Stimuli}

The digital signal for the stimuli was produced in the same way as in the previous subsection. However, modulation frequencies $f_{\mathrm{m}}$ of $4,8,16,32$, and $64 \mathrm{~Hz}$ were used at a fixed modulation index $m$ of 1.0 .

\section{B. Results and discussions}

In Fig. 5, the upper thresholds as well as the modulation period are plotted as a function of modulation frequency $f_{\mathrm{m}}$. The modulation period is the duration for one cycle of the modulation or $1 /$ $f_{\mathrm{m}}$. Although there is a slight increase in the upper threshold from $f_{\mathrm{m}}$ of $4 \mathrm{~Hz}$ to $8 \mathrm{~Hz}$, the upper threshold decreases as $f_{\mathrm{m}}$ increases to $16 \mathrm{~Hz}$ and over. For example, the upper threshold for $f_{\mathrm{m}}$ of 4 and 8 $\mathrm{Hz}$ is roughly $360 \mathrm{~ms}$, while the upper threshold for $f_{\mathrm{m}}$ of $64 \mathrm{~Hz}$ is $135 \mathrm{~ms}$.

According to the $t$-test, the difference between the upper threshold for the $f_{\mathrm{m}}$ of $4 \mathrm{~Hz}$ and that for the $f_{\mathrm{m}}$ of $8 \mathrm{~Hz}$ is not significant $(t(3)=1.542 ; p>0.1)$. An analysis of variance on the whole data indicates that the effect of $f_{\mathrm{m}}$ is significant $(F(4,12)=92.99$;

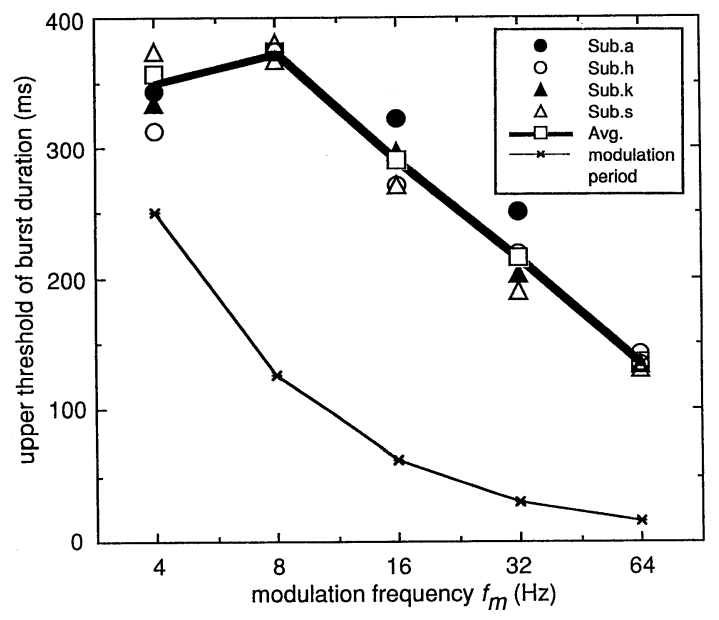

Fig. 5 Upper threshold of burst duration for amplitude-modulated broadband noises as a function of modulation frequency $f_{\mathrm{m}}$ at a modulation index $m$ of 1.0 . $p<0.001)$. However, no significant effect of subject was found $(F(3,12)=1.252 ; p>0.1)$.

Figure 5 shows that the upper threshold declined more slowly than the modulation period as $f_{\mathrm{m}}$ increased. Compared with the upper threshold and the modulation period, this slower decrease indicates that the number of modulation cycles per stimulus source for the upper threshold is dependent on the $f_{\mathrm{m}}$. Therefore, it is unlikely that the subjects assessed the continuity owing to the number of modulation cycles.

The results obtained in subsections 3.1. and 3.2. show systematical variation in the upper threshold with $m$ and $f_{\mathrm{m}}$. Compared with the upper threshold of $72 \mathrm{~ms}$ for unmodulated broadband noise $(m=0.0)$, the factor of the increase in the upper threshold for amplitude-modulated broadband noise can be attributed to the amplitude modulation.

This dependence of the upper threshold on $m$ and $f_{\mathrm{m}}$ can be explained in terms of the perceived strength of the fluctuation and that of the roughness, which are elicited by modulation. As Fastl (1990) reviewed, the following properties of the fluctuation and the roughness have been investigated: (1) The strength of the fluctuation and that of the roughness increase as $m$ increases. (2) A noncritical transition in perception occurs at a modulation frequency $f_{\mathrm{m}}$ of about $20 \mathrm{~Hz}$, from the fluctuation that is elicited at a lower $f_{\mathrm{m}}$ to the roughness at a higher $f_{\mathrm{m}}$. (3) The strength of the fluctuation decreases as the $f_{\mathrm{m}}$ heightens. (4) Instead, the strength of the roughness reaches a maximum near $f_{\mathrm{m}}$ of $70 \mathrm{~Hz}$. Qualitatively, the upper threshold and the fluctuation strength demonstrate similar tendencies in response to variation in the modulation index $m$ and in the modulation frequency $f_{\mathrm{m}}$. The transition frequency of $20 \mathrm{~Hz}$ is close to the $f_{\mathrm{m}}$ of $16 \mathrm{~Hz}$ where the upper threshold starts to decline as $f_{\mathrm{m}}$ heightens. These dependences of the strength on $f_{\mathrm{m}}$ and $m$ indicate relevance to those of the upper threshold without ruling out the notion; temporal variation in the perceived strength of amplitude modulation provides a cue to motion detection.

Based on this hypothesis, it is plausible that the fluctuation elicited by amplitude modulation interferes with the detection of abrupt variation in amplitude due to successive presentation of stimuli. With respect to the lowering of the fluctuation for high modulation frequencies, the detection of the 
fluctuation or loudness follows the short-time averaging of the amplitude (de Boer, 1966). The averaged version of the amplitude modulation introduced in the stimuli may hamper the discrimination of the change in the amplitude caused by successive presentations. The ambiguity of the perception for the amplitude modulation may cause continuous apparent motion for amplitudemodulated broadband noise even at longer burst durations relevant to the variation in the upper threshold mediated by $m$ and $f_{\mathrm{m}}$. This explanation is consistent with the following hypotheses: (1) temporal variation in amplitude is used as a cue to motion (Strybel and Neale, 1994), and (2) the spatial detector uses the output from the loudness detector (Rutland-Reinhardt, 1992). Notion (1) can be supported by the ambiguity of the perception between the amplitude modulation introduced to the stimuli and the variation in the amplitude originating from the successive presentation. Notion (2) may be evidenced by the delay of the decision for the position of the posterior stimulus, reflected in the increase in the upper threshold.

\subsection{Frequency Dependence for Tones}

It should be remembered that the experiment in the foregoing subsections 3.1 and 3.2 were conducted by using amplitude-modulated broadband noise that contained full bands of frequency component. The modulation frequency $f_{\mathrm{m}}$ does not specify the band of frequency component, but the amplitude modulation. With such frequency component coverage, the characteristics of the upper threshold by a specific band of frequency was not allowed to be considered. To investigate the effect of frequency on the upper threshold, we conducted further experiments to test tones, which can be specified by frequency.

\section{A. Stimuli}

A sinusoidal signal produced in the computer was used as the tone, as follows

$$
A \sin \left(2 \pi f i / f_{\mathrm{S}}\right)
$$

where $A$ is the amplitude, $f$ is the frequency, $f_{\mathrm{s}}$ is the sampling frequency (fixed at $24 \mathrm{kHz}$ ), and $i$ is the $i$-th order. Consequently, the tone

$$
A \sin (2 \pi f t)
$$

is presented by the loudspeakers, where $t$ is the time. In this experiment, frequencies of $250,500,1,000$,

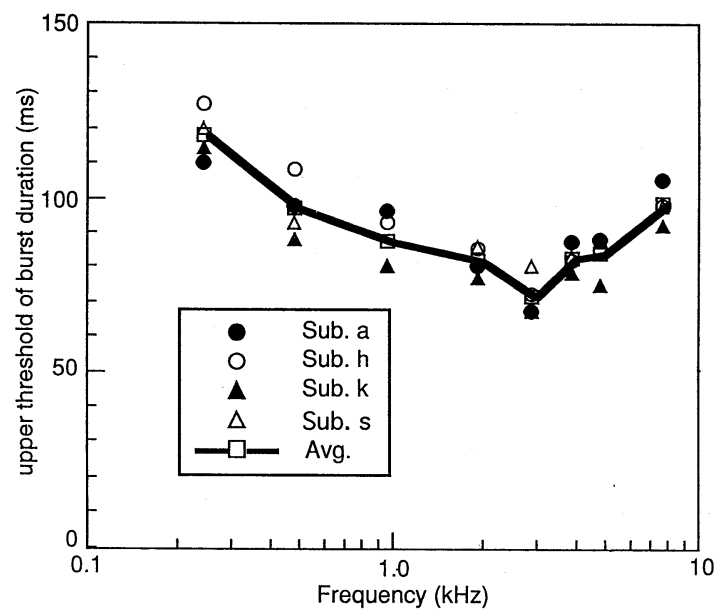

Fig. 6 Upper threshold of burst duration for tones as a function of frequency $f_{\mathrm{m}}$.

2,000, 3,000, 4,000, 5,000 and $8,000 \mathrm{~Hz}$ were tested.

B. Results and discussion

The upper thresholds for tones are plotted as a function of frequency in Fig. 6. They display a concave function with a minimum of $71 \mathrm{~ms}$ at a frequency of $3,000 \mathrm{~Hz}$. The upper threshold for $250 \mathrm{~Hz}$ is $118 \mathrm{~ms}$, and that for $8,000 \mathrm{~Hz}$ is $98 \mathrm{~ms}$. An analysis of variance of the data indicates that the effect of the frequency is significant $(F(7,21)=$ $24.22 ; p<0.001)$. However, this increase in the upper threshold compared with that for $3,000 \mathrm{~Hz}$ is inferior to the increase in the upper threshold for amplitude-modulated broadband noises (e.g., 290 ms for $m=1.0$ ) compared with that for unmodulated broadband noise of $72 \mathrm{~ms}$. The frequency dependence of the upper threshold can be explained in terms of the sensibility to the variation in intensity. According to Riesz (1928), the just-noticeable level difference is least in the middle frequencies of 2,000 to $4,000 \mathrm{~Hz}$ and becomes less sensitive for lower and higher frequencies. This property is also consistent with the hypothesis that the ambiguity of abrupt variation in the loudness provides a cue for detecting the succession.

The upper threshold for $3,000 \mathrm{~Hz}(71 \mathrm{~ms})$ is nearly equal to that for unmodulated broadband noise of $72 \mathrm{~ms}$. With regard to the proximity between the upper thresholds, it can be hypothesized that the frequency component for the shortest upper threshold contained in the stimuli principally contributes to the determination of the upper threshold. Occa- 


\section{KINOSHITA and S. AOKI : DURATION FOR CONTINUOUS APPARENT MOTION}

sionally, the upper threshold for unmodulated broadband noise was reconciled with that for the $3,000-\mathrm{Hz}$ tone, for which the upper threshold is minimum over frequency.

\subsection{Bandwidth Dependence for Bandpass Noises}

In this subsection, we describe our investigation of several bands of bandpass noise in an attempt to confirm our hypotheses for dependences of the upper threshold obtained in subsections 3.1 through 3.3. We expected to assess the notion that the composition of the frequency component would reconcile the minimum upper threshold over the frequency range in the stimuli. The bandwidth variation can also decompose the cues used to judge the continuous motion in a frequency domain in a different way from than in subsection 3.3.

\section{A. Stimuli}

A pseudo-random signal produced by the computer was used as the bandpass noise. The bandwidth was adjusted by means of the cutoff frequencies of the low- and high-pass filters. To test 1/12-, 1/6-, 1/3-, 1/2-, 1-, 2- and 3-octave bands of noise, each with a center frequency of $1,000 \mathrm{~Hz}$, the combination of cut-off frequencies for the low- and high-pass filters were set, respectively, at 1,030 and 972, 1,060 and 944, 1,120 and 891, 1,190 and 841, 1,410 and 707, 2,000 and 500, and 2,830 and $354 \mathrm{~Hz}$.

B. Results and discussion

Figure 7 shows the upper threshold for the bandpass noises as a function of bandwidth. For com-

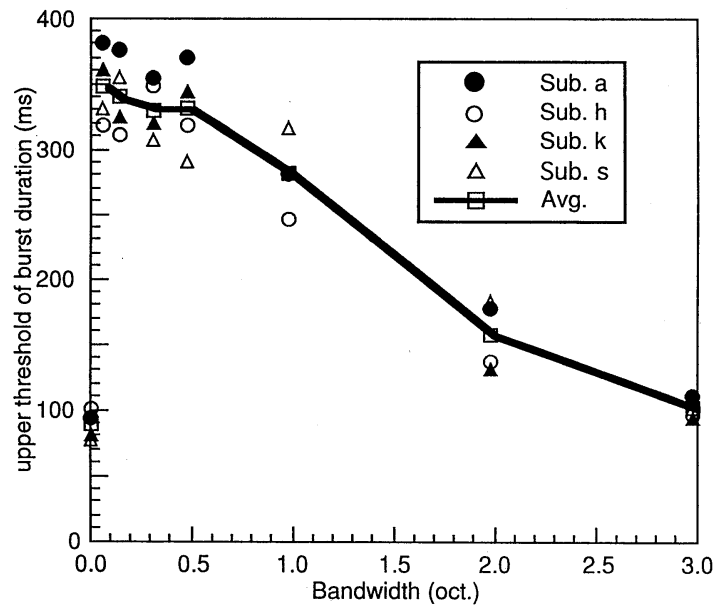

Fig. 7 Upper threshold of burst duration for bandpass noises as a function of bandwidth at a center frequency $f_{\mathrm{c}}$ of $1,000 \mathrm{~Hz}$. parison, the upper threshold for a $1,000-\mathrm{Hz}$ tone in the above mentioned experiment is also shown as that at 0.0 octaves. An analysis of variance indicates a significant effect of bandwidth on the upper thresholds $(F(6,18)=42.57 ; p<0.001)$. The upper threshold decreases as the band broadens and seems to converge with that for a broadband noise of $72 \mathrm{~ms}$. The upper threshold for the 1/12-octave band of the noise was $347 \mathrm{~ms}$, almost fivefold that for the broadband noise, and the upper threshold for the 3-octave band of noise was $101 \mathrm{~ms}$. However, the upper threshold for the $1,000-\mathrm{Hz}$ tone of $87 \mathrm{~ms}$ was far lower than that for the 1/12-octave band of the noise, while a slight difference was obtained between the upper threshold for the $1,000-\mathrm{Hz}$ tone and that for unmodulated broadband noise. In addition, the effect of the bandwidth on the upper thresholds for the bands narrower than one octave was insignificant $(F(3,9)=0.434 ; p>0.25)$.

Unlike the hypothesis in the previous subsection, it is implausible that the upper threshold can be reconciled with the minimum upper threshold over the frequency component in the stimulus. There is a prominent difference between the upper threshold for a pure tone and that for $1 / 12$ octave band noise, whereas there is a gradual decrease in the upper threshold caused by the extention of the bandwidth.

It is more probable that amplitude modulation is an essential factor contributing to a considerable increase in the upper threshold of the burst duration for continuous apparent motion. The fluctuation elicited from the envelope modulation may cause the increase in the upper threshold. In fact, the envelope is not unmodulated in tones, while it is in the bandpass noises. As can be seen from the waveforms shown in Figs. 8(a) through (g), the number of local maxima in the envelope increases as the bandwidth extends (Fastl, 1990). For example, the numbers of the local maxima per second are 10 for $1 / 12$ octave and 16 for $1 / 2$ octave. Although direct comparison with the results in subsection 3.1 and 3.2 is not feasible due to the random variation in the envelope, a qualitative interpretation for the dependence of the upper threshold on bandwidth is possible. As the number of local maxima increases or the bandwidth widens, the upper threshold decreases. Even the dependence on the bandwidth can be explained based on the assumption that the fluctuation and the roughness retard resolution of the sound location. This delay may cause the 

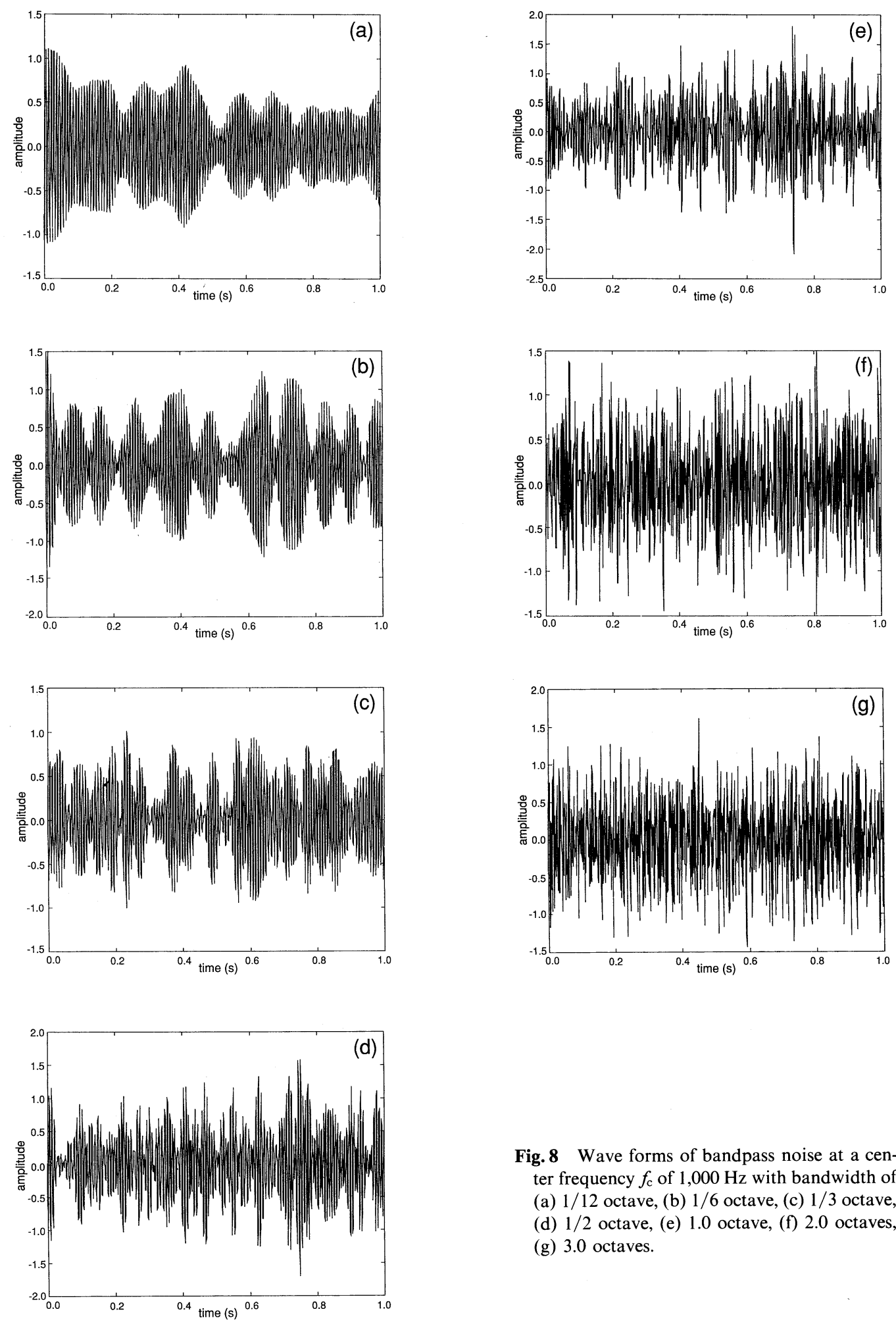

Fig. 8 Wave forms of bandpass noise at a center frequency $f_{\mathrm{c}}$ of $1,000 \mathrm{~Hz}$ with bandwidth of (a) $1 / 12$ octave, (b) $1 / 6$ octave, (c) $1 / 3$ octave, (d) $1 / 2$ octave, (e) 1.0 octave, (f) 2.0 octaves, (g) 3.0 octaves. 
increase in the upper threshold for the narrow bands of noise.

The explanation can be applied to the reduction in the upper threshold for broader bands of noise, which exhibits rapid variation in amplitude. In fact, the extent of the upper threshold for bandpass noise narrower than $1 / 2$ octave (330 to $350 \mathrm{~ms}$ ) is covered by that for amplitude-modulated broad bandnoise for $f_{\mathrm{m}}$ lower than $16 \mathrm{~Hz}$ (290 to $\left.350 \mathrm{~ms}\right)$. The frequency modulation at a modulation frequency $f_{\mathrm{m}}$ below $16 \mathrm{~Hz}$ (or the number of the local maxima) might be responsible for the sluggish variation in the upper threshold for bands of noise narrower than $1 / 2$ octave. Below the modulation frequency $f_{\mathrm{m}}$ of $16 \mathrm{~Hz}$, a saturating increase, in relation to the result in subsection 3. 2, in the upper threshold on the modulation frequency $f_{\mathrm{m}}$ for amplitudemodulated broadband noise was observed.

\section{GENERAL DISCUSSION}

We investigated not only the dependence of the upper threshold on the modulation index and on the modulation frequency, but also that on the frequency and on the bandwidth. These relations can be attributed to the temporal variation in envelope.

The reader may recall the minimum integration time, which is required for localization with best accuracy. It is one of the scales for localization accuracy for a moving source (Chandler and Grantham, 1992). The minimum integration time for a 1/3-octave band noise source of bandpass noises of $420 \mathrm{~ms}$ is comparable to the upper thresholds for the stimuli of $350 \mathrm{~ms}$. Chandler and Grantham (1992) presumed that the increase in the minimum integration time was due to modulation of the envelope. However, the minimum integration time exhibits a property different from that for the upper threshold. The minimum integration time is not considerably dependent on the source, and its extent is confined within 290 to $420 \mathrm{~ms}$ for stimuli located in front of the subject, which differs from the case with the upper threshold. For example, the minimum integration time for tones and that for the unmodulated broadband noise are both almost $300 \mathrm{~ms}$, which considerably exceed the upper threshold for $3,000-\mathrm{Hz}$ tone $(71 \mathrm{~ms})$ or that for unmodulated broadband noise $(72 \mathrm{~ms})$. In summing up these properties of the minimum integration time, the following explanation cannot be ruled out: the motion of the unmodulated stimulus is detected before judgement of each position of stimulus, whereas the motion of the modulated stimulus is detected after such judgement. Therefore, continuous apparent motion may be judged after detection of variation in the localized position of the stimulus over time for the modulated stimulus, but not for the unmodulated stimulus.

Insofar as unmodulated stimuli are concerned, this explanation does not exclude the assumption that the judgement of motion direction is processed by different mechanisms for production of apparent motion (Strybel 1994, Strybel and Neales 1994). The judgement of motion direction is usually much more time-consuming than the detection of motion and requires at least the perception of sound positions at the initial and the later stages. In contrast, motion can be detected without need for judgement of positions.

As mentioned in section 3 , the results obtained in the current paper may be relevant to the perception for amplitude modulation. It is accepted that the dependence of the fluctuation strength on the modulation index $m$ and the modulation frequency $f_{\mathrm{m}}$ can be modelled as smoothing or integration of the power over time (Fastl, 1990). Presumably, the interference between motion detection and the fluctuation contributes to the spatial smoothing, which leads to the increase in the upper threshold.

Such explanations are consistent with the twostage hypothesis (Rutland-Reinhardt, 1992) that explains the processing mechanism in analyzing motion; output from channels for sound-level change stimulate movement channels. The soundlevel and the movement detectors are assumed to receive the temporal variation in loudness and that in position, respectively; that is, the fluctuation and the motion. The increase in the upper threshold can be more plausibly explained in terms of the perception of amplitude modulation, rather than by the temporal variation in modulation itself or by filtering.

\section{SUMMARY}

The upper thresholds of burst duration that produced continuous apparent motion were measured when stimuli were successively presented using three loudspeakers arranged in the horizontal plane. In this experiment, amplitude-modulated broadband noises, tones and bandpass noises were tested as stimuli. The upper thresholds for the stimuli 
tested were usually longer than that for an unmodulated broadband noise. The upper threshold was observed both to increase with an increase in the modulation index and to decrease with an increase in the modulation frequency. For tones, the upper threshold displayed a concave function of frequency with a minimum at a frequency of $3,000 \mathrm{~Hz}$. For bandpass noises, the upper threshold decreased as the bandwidth broadened. Through these findings, this study offers evidence that motion detection can be processed subsequent to perception of variation in loudness elicited by amplitude modulation. The results are consistent with the two-stage hypothesis that explains the mechanisms underlying the motion detection that follows processing of amplitude modulation.

\section{REFERENCES}

Briggs, R. and Perrott, D. R. (1972). "Auditory apparent movement under dichotic listening conditions," J. Exp. Psychol. 92, 83-91.

Burtt, H. E. (1917). "Auditory illusions of movement -A preliminary study,” J. Exp. Psychol. 2, 63-75.

Chandler, D. W. and Grantham, D. W. (1992). "Minimum audible movement angle in the horizontal plane as a function of stimulus frequency and bandwidth, source azimuth, and velocity," J. Acoust. Soc. Am. 91, 16241636.

de Boer, E. (1966). "Intensity discrimination of fluctuating signals," J. Acoust. Soc. Am. 40, 552-560.

Fastl, H. (1990). "Fluctuation Strength," in Psychoacoustics, E. Zwicker and H. Fastl, Eds. (Springer-Verlag, Berlin, 1990), Chap. 10, pp. 222-236.

Forrest, T. and Green, D. M. (1987). "Detection of partially filled gaps in noise and the temporal modulation transfer function," J. Acoust. Soc. Am. 82, 1933-1943.

Hisata, T. (1934). "On the apparent moving phenomenon in auditory modality," Psychol. Stud. 9, 367-400 (in Japanese).

Kinoshita, I. and Aoki, S. (1993). "Effects of source signals on the perception of continuity for sequentially reproduced sounds," Tech. Rep. Hear. Acoust. Soc. Jpn. H93-2, 1-8 (in Japanese).

Korte, A. (1915). "Kinematoskopische Untersuchungen," Z. Psychol. 72, 193-206.

Mizushima, K., Nakanishi, S., and Morimoto, M. (1994). "Continuity of a moving sound image caused by succes- sive signals from two discretely located loudspeakers," J. Acoust. Soc. Jpn. (E) 15, 179-188.

Nakajima, T., Tamaribuchi, K., and Saito, S. (1990). "Perception of the motional image induced from distributed sound sources excited sequentially," Tech. Rep. Hear. Acoust. Soc. Jpn. H-90-6, 1-6 (in Japanese).

Nakanishi, S., Morimoto, M., and Mizushima, K. (1994). "Relation between continuity of a moving sound image by successive presentation of signals and temporal detection," Proc. Spring Meet. Acoust. Soc. Jpn. 1-4-18 (in Japanese).

Riesz, R. R. (1928). "Differential intensity sensibility of the ear for pure tones," Phys. Rev. 31, 867-875.

Rutland-Reinhardt, A. H. (1992). "Changing-loudness aftereffect following simulated movement: Implications for channel hypotheses concerning sound level change and movement," J. Gen. Psychol. 119, 113-121.

Scholz, W. (1924). "Experimentalle Untersuchungen über die phänomenale Größe von Raumstrecken, die durch Sukzessiv-Darbietung zweier Reize begrenzt werden," Psychol. Forsch. 5, 219-272.

Shailer, M. J. and Moore, B. C. J. (1983). "Gap detection as a function of frequency, bandwidth, and level," J. Acoust. Soc. Am. 74, 467-473.

Strybel, T. Z., Manligas, C. L., and Perrott, D. R. (1989). "Auditory apparent motion under binaural and monaural listening conditions,” Percept. Psychophys. 45, 371377.

Strybel, T. Z., Manligas, C. L., Chan, O., and Perrott, D. R. (1990). "A comparison of the effects of spatial separation on apparent motion in the auditory and visual modalities," Percept. Psychophys. 45, 371-377.

Strybel, T. Z., Witty, A. M. and Perrott, D. R. (1992). "Auditory apparent motion in the free field: The effects, of stimulus duration and separation," Percept. Psychophys. 52, 139-143.

Strybel, T. Z. (1993). "Auditory apparent motion in the horizontal and median planes," J. Acoust. Soc. Am. 94, 1778.

Strybel, T. Z. (1994). "The role of amplitude modulation in auditory motion,” J. Acoust. Soc. Am. 95, 3004.

Strybel, T. Z. and Neale, W. N. (1994). "The effect of burst duration, interstimulus onset interval, and loudspeaker arrangement on auditory apparent motion in the free field," J. Acoust. Soc. Am. 96, 3463-3475.

Stumpf, E., Toronchuk, J. M. and Cynader, M. S. (1992). "Neurons in çat primary auditory cortex sensitive to correlates of auditory motion in three-dimensional space," Exp. Brain Res. 88, 158-168 